\title{
GESTÃO DE SUPRIMENTOS NA FARMÁCIA HOSPITALAR PÚBLICA
}

\section{ADMINISTRATION OF SUPPLIES IN THE PHARMACY HOSPITAL PUBLIC}

\author{
Luciane Dalarmi ${ }^{1}$ \\ 1 - Farmacêutica Responsável pela Farmácia Hospitalar do Hospital Regional Infantil em Campo Largo - Paraná \\ REC: $11 / 09$ AC: $12 / 09$
}

\begin{abstract}
Resumo
A gestão de suprimentos em hospitais públicos vem se tornando uma tarefa das mais complexas e vai além da burocracia. Administrar, é gerir com ênfase nos recursos humanos, no conhecimento teórico e nos processos. Deste último encontra-se o ato de suprir, adequadamente, o ambiente hospitalar com materiais adequados que garantam a qualidade, a produtividade, a satisfação dos pacientes e a prestação de serviços pela equipe hospitalar. Este estudo visa analisar a complexidade da Gestão de Suprimentos na Gestão Hospitalar Pública, levantando conceitos, identificando atividades e benefícios de uma gestão voltada para o processo de suprimento hospitalar com competência e responsabilidade. Conhecendo cada detalhe desse processo, seu desenvolvimento, suas limitações e as formas de conduzílo minimizando custos sem ferir a excelência na prestação de serviços é dever de todo gestor. Neste contexto, discute-se a gestão de estoque da farmácia hospitalar do Hospital Regional Infantil Doutor Waldemar Monastier, um exemplo. Os estudos que versam sobre o tema enfocam questões de aquisição, suprimento, alinhamento, além de uma visão crítica da logística de suprimentos em hospitais públicos. Desta forma, a metodologia da pesquisa bibliográfica acerca do referencial teórico que aborda o tema, norteou esse estudo que não tem a pretensão de desvelar esse conteúdo, pois além de complexo é, ainda, alvo de investigação. Á título de conclusão convida aos gestores farmacêuticos, atuais e futuros, a darem continuidade à práxis, na busca da eficácia da Gestão Hospitalar em Suprimentos.
\end{abstract}

Palavras chaves: Gestão de suprimentos hospitalar, logística.

Abstract

Administer became one of the most complex task and goes beyond the bureaucracy. Administer is managed with an emphasis on human resources, intellectual capital and processes. Among the latter is to meet adequately the hospital with appropriate materials to ensure the quality, productivity, patient satisfaction and service delivery by the hospital staff. This study analyzes the complexity of Supply Management in Public Hospital, lifting concepts, identifying activities and benefits of management focused on the procurement process from hospital with competence and responsibility. Knowing every detail of this process, its development and ways to lead you minimize costs without hurting the excellence in service is the duty of every manager. In this context, discusses the inventory management of hospital pharmacies Regional Hospital Infantil Dr. Waldemar Monastier, an example. Studies that focus on the subject focus on issues of acquisition, resupply, and alignment, beyond a critical view of the logistics supply in public hospitals. Thus, the methodology of the research literature on the theoretical framework that addresses the topic, guided this study does not attempt to uncover such content, as well as the complex is still under investigation. In conclusion, this study calls for pharmacist managers, present and future, to follow up the practice, in the pursuit of effectiveness of the Hospital Management in Supply.

Keywords: Management of Hospital Supplies, Logistics. 


\section{INTRODUÇÃO}

A instituição hospitalar abriga a farmácia hospitalar, cujo objetivo é garantir o uso seguro e racional dos medicamentos prescritos pelo profissional médico, além de responder à demanda das necessidades de medicamentos e de produtos para saúde dos pacientes hospitalizados.

Para tanto, a farmácia hospitalar mantém sob sua guarda os estoques desses produtos que são caracterizados por ciclos de demandas e de ressuprimentos. Estes com flutuações significativas e altos graus de incerteza, fatores críticos diante da necessidade de manter medicamentos em disponibilidade na mesma proporção da sua utilização (NOVAES;GONÇALVES; SIMONETTI, 2006). A grande variedade de itens utilizados num hospital aliada ao risco por desabastecimento pode promover estoques em excesso, o que significa recursos financeiros imobilizados, espaços mal utilizados, consumo excessivo de energia e risco de descarte dos materiais (PAULUS JR, 2005).

A preocupação com a logística hospitalar vem crescendo bastante, pois dela depende, entre outros setores, o abastecimento de todos os pontos de distribuição de medicamentos e materiais médico-hospitalares dentro do hospital, independente do valor. A logística é vital não só para o funcionamento dos hospitais, mas para todas as organizações, principalmente aquelas que são obrigadas a trabalhar com estoques com um grande volume de suprimentos (YUK; KNEIPP; MAEHLER, 2007). Nessa óptica, o gerenciamento de estoques norteia a redução dos custos gerados pelo mesmo através de técnicas adequadas inviabilizando a deterioração da qualidade do serviço de saúde.

A lentidão nos processos administrativos presenciado nos serviços de saúde no Brasil é apontada como problema crônico, gerando formalidade nas comunicações, excessiva burocracia, apresentando serviços que em muitas vezes não satisfazem os prestadores de serviço.

Após a constatação do atual cenário, a pergunta que se faz é: qual é a saída? Senge (1995), na tentativa de responder a esses e outros tantos questionamentos, conclui: "ninguém possui as respostas para essas perguntas" (p. 5). O que resta é continuar tentando, pesquisando, aliando teoria e prática na intenção de buscar soluções, inventar propostas e alternativas, a fim de otimizar o processo de aquisição, estoque, distribuição de medicamentos e produtos para saúde.

Objetivou-se com este trabalho analisar a complexidade da gestão de suprimentos na farmácia hospitalar, apontando a logística de material e medicamento e as dificuldades da gestão destes em hospitais públicos.

\section{LOGÍSTICA}

Segundo Ballou (2006), "logística é o processo de planejamento, implantação e controle do fluxo eficiente de mercadorias, serviços e das informações relativas desde o ponto de origem até o ponto de consumo com o propósito de atender às exigências dos clientes". A logística hospitalar, entretanto, abrange desde as infra-estruturas existentes, a organização e as pessoas, os processos e os sistemas de informação de suporte.

O mais importante aspecto da vida hospitalar está intimamente ao cuidado com o paciente, até a sua possível recuperação. Assistência médico-hospitalar satisfatória será o mínimo que a administração por meio de um complexo conjunto de recursos materiais e humanos deve proporcionar ao paciente (Maudonnet 1988). 
O perfeito entendimento da cadeia de abastecimento tem sido reconhecidamente um fator de vantagem competitiva para as organizações que efetivamente entendem o seu papel estratégico. A cadeia de abastecimento corresponde ao conjunto de processos requeridos para obter materiais, agregar-Ihes valor de acordo com a concepção de pacientes e consumidores e disponibilizar os produtos para o lugar (onde) e para a data (quando) que os pacientes e consumidores os desejarem. Nos últimos anos este conceito sofreu evoluções, onde a cadeia de abastecimento apresenta uma visão mais ampla que a cadeia logística, a qual se limita a obtenção e movimentação de materiais e à distribuição física de produtos (Bertaglia, 2005).

\subsection{Logística de medicamentos e material médico hospitalar no Estado do Paraná}

A assistência farmacêutica no contexto do SUS é uma ação de saúde pública e parte integrante do sistema de saúde. "Na Política Nacional de Medicamentos a assistência farmacêutica está definida como um grupo de atividades relacionadas com o medicamento destinadas a apoiar as ações de saúde demandadas por uma comunidade. Envolve o abastecimento de medicamentos em todas e em cada uma de suas etapas constitutivas, a conservação e controle de qualidade, a segurança e a eficácia terapêutica dos medicamentos, o acompanhamento e avaliação da utilização, a obtenção e a difusão de informação sobre medicamentos e a educação permanente dos profissionais de saúde, do paciente e da comunidade para assegurar o uso racional de medicamentos" (BRASIL, 1998).

A Política Nacional de Medicamentos, publicada em 1998 por meio da portaria GM/ MS $n^{\circ} 3.916$, tem como principal finalidade garantir segurança, eficácia e qualidade dos medicamentos, promover o uso racional dos mesmos além de prover o acesso da população àqueles medicamentos considerados essenciais. Esta política apresenta um conjunto de diretrizes para alcançar tais objetivos, que incluem como prioridade "a revisão permanente da Relação Nacional de Medicamentos Essenciais - RENAME, a reorientação da assistência farmacêutica, a promoção do uso racional de medicamentos e a organização das atividades de Vigilância Sanitária". Mais especificamente, a reorientação da Assistência Farmacêutica se encontra fundamentada na "descentralização da gestão, na otimização e eficácia do sistema de distribuição no setor público e no desenvolvimento de iniciativas que possibilitem a redução nos preços dos produtos" (BRASIL, 1998).

A Assistência Farmacêutica representa hoje um setor de grande impacto financeiro no âmbito das Secretarias Estaduais de Saúde, pela crescente demanda por medicamentos. É constituída por três componentes; Componente Básico, Estratégico e Especializado da Assistência Farmacêutica.

Esta abrange as atividades de seleção, programação, aquisição, armazenamento, distribuição e acompanhamento da utilização de medicamentos. Além de ser uma atividade relevante nas ações de saúde, deve ser contemplada com a adequação da necessidade, segurança, efetividade e qualidade da terapia, promovendo o uso racional dos medicamentos e contribuindo para a melhoria das condições de vida e de saúde da população.

A Secretaria de Estado de Saúde do Paraná tem como missão sustentar uma política de construção permanente de um modelo de saúde que cumpra a função social de promoção, prevenção e atenção à saúde, contribuindo para a melhoria da qualidade de vida da população paranaense. Um de seus objetivos é prover o Sistema Único de Saúde de insumos (medicamentos, imunobiológicos, sangue e hemoderivados), em quantidade adequada e com qualidade (www.saude.com.gov.br, 2010).

Visão Acadêmica, Curitiba, v.11, n.1, Jan. - Jun./2010 - ISSN 1518-5192 
Dentro deste contexto, a distribuição das medicações nas instituições de saúde públicas no estado do Paraná é centralizada no Centro de Medicamentos do Paraná- CEMEPAR. CEMEPAR é uma unidade subordinada à Superintendência de Gestão de Sistemas de Saúde, tendo como propósito a garantia do acesso da população aos medicamentos dos programas oferecidos pelo Ministério da Saúde e pela Secretaria de Estado da Saúde do Paraná - SESA/ PR.

O CEMEPAR é responsável pela implantação e implementação da Política de Assistência Farmacêutica no estado, em consonância com o Plano Estadual de Saúde e com as diretrizes estabelecidas para este setor pelo Ministério da Saúde. Este disponibiliza medicações para 22 hospitais e unidades de saúde no Estado do Paraná. Entre estes hospitais, diariamente realizam-se remanejamentos por falta de medicações, aumento de consumo ou equívocos de dimensionamento.

Dividida em vinte e duas Regionais de Saúde, a estrutura organizacional da SESA é utilizada pelo CEMEPAR para efetivar a distribuição e dispensação dos medicamentos aos pacientes dos hospitais públicos. Essas Regionais de Saúde constituem parte integrante na operacionalização do ciclo da Assistência Farmacêutica. O Plano Estadual de Assistência Farmacêutica, através do CEMEPAR, descreve as atividades e ações de cada programa da Assistência Farmacêutica, a fim de atingir os objetivos preconizados na Política Nacional de Assistência Farmacêutica.

Torna-se de extrema importância para o gestor farmacêutico o entendimento destas políticas e responsabilidades de cada segmento da assistência farmacêutica, não apenas no que diz respeito ao abastecimento de medicamento em nível hospitalar, mas também no encaminhamento de pacientes nos respectivos programas de aquisição de medicamentos do Estado (toxoplasmose congênita, doença hemolítica do recém nato, análogos de insulina, Paraná sem dor, fibrose cística).

No que se refere ao abastecimento de material médico hospitalar, alimentos, materiais de higiene, limpeza, entre outros materiais de consumo em instituições públicas é responsabilidade do DELS - Departamento de Logística de Saúde.

As solicitações de material ao DELS, realizado por pedidos trimestrais e, eventualmente, pedidos emergenciais, possuem inúmeras limitações. Raramente são atendidos integralmente ocorrendo, portanto, ruptura no estoque. Cabe ao gestor farmacêutico cuidar destas rupturas ocasionadas pela falha de entrega para que a assistência dos pacientes não seja prejudicada.

\section{COMPRAS}

No setor público a aquisição de material deve ser realizada por meio de um processo formal chamado de licitação, desenvolvido conforme os preceitos estabelecidos para esse fim e com o objetivo de atender às necessidades da organização quanto à compra de produtos, bens ou serviços. Diante disto, uma boa especificação técnica dos materiais antes da sua aquisição minimiza os problemas decorrentes do não atendimento deste produto às especificidades do procedimento no qual ele será utilizado.

As licitações no Brasil estão regulamentadas pela Lei 8.666 de 21 de junho de 1993, atualizada pelas leis 8.883 de 8 de junho de 1994 e 9.648 de 27 de maio de 1998. Todo administrador público deve conhecê-las. As licitações possuem determinados princípios básicos que devem ser observados para que ao final do processo este seja válido e atenda aos objetivos do processo de compra. Segundo a legislação vigente, as empresas que participam de determinadas modalidades de licitação devem apresentar documentos que forneçam provas da capacidade jurídica e de regularidade fiscal da empresa. Tanto a revogação quanto 
a anulação levam ao mesmo resultado, ou seja, a suspensão do trâmite. A revogação é um ato que a administração pode tomar no interesse público, desde que justifique devidamente a sua necessidade, podendo revogar determinados itens ou mesmo todo o processo. A anulação é determinada por alguma ilegalidade existente no processo que obriga a sua interrupção. O julgamento é o ato que seleciona a proposta mais vantajosa, que atenda aos critérios especificados no edital. As modalidades de licitação são definidas pelos limites de valores fixados pela legislação, podendo ocorrer através de: convite; tomada de preços; concorrência; leilão; concurso ou pregão.

\section{GESTÃO DE SUPRIMENTOS NA FARMÁCIA HOSPITALAR PÚBLICA}

Santich (1995, p.52-53) apresenta como principais problemas dos serviços farmacêuticos brasileiros, no setor público, "o desabastecimento cíclico e quase permanente da maioria dos produtos necessários; uso irracional (prescrição sem base científica); consumo descontrolado; desperdício pelo manuseio incorreto; perdas de quantidades significativas pelo vencimento da validade; perdas por dispensação inadequada; perdas por distribuição inadequada; sistema de compras deficiente; administração e gestão deficiente em todo o processo; insatisfação crescente dos clientes internos e externos; ambiente de trabalho pouco amigável; roubo, corrupção e descompromisso." Estes problemas registrados por Santich são, em sua essência, decorrentes da cadeia de suprimento e remetem a um aperfeiçoamento do sistema de planejamento e controle do processo produtivo.

O abastecimento de medicamentos constitui, no serviço público, uma prática que recebe influência de vários fatores sociais, culturais e biológicos. Por isso, deve pautar-se numa visão científica que contemple esta complexidade de fatores. Neste sentido, o "planejamento" constitui um elemento essencial ao gerenciamento do serviço de abastecimento. Constata-se, nas rotinas dos serviços de saúde, que a integração sistêmica desses componentes está longe de ser uma realidade. Isso ocorre por dois fatores básicos. Por um lado, na maioria das vezes, as decisões políticas suplantam as técnicas e, além disso, os procedimentos operacionais são burocráticos e conservadores. Por outro, as informações técnicas existentes são pouco consideradas no momento de planejamento, de modo que o pensamento sobre a qualidade fica num nível de acomodação em face das dificuldades conjunturais.

A gestão da cadeia de suprimento é um elemento de destaque na gestão dos serviços de saúde, tendo esta uma fundamentação técnico-científica centrada nos parâmetros epidemiológicos da comunidade atendida e nas prioridades demandadas a partir das necessidades sanitárias. Nesse aspecto, Laporte (1989, p.57-72) destaca que as informações de natureza epidemiológica são essenciais para os procedimentos de seleção de medicamentos, definição das prioridades sanitárias, estruturação do sistema, definição dos recursos financeiros e humanos, entre outros aspectos.

Dentro dessa lógica sistêmica dos serviços de saúde e da assistência farmacêutica, é imprescindível responder-se aos seguintes questionamentos: Em que condições são adquiridos os medicamentos para os serviços públicos ambulatoriais de saúde? Quais os determinantes das quantidades a serem adquiridas por estes serviços? Como se processa sua distribuição na rede pública? As respostas a tais questionamentos poderão subsidiar a construção de um processo racional de aquisição, armazenagem e distribuição de medicamentos no serviço público de saúde, apresentando como conseqüência a otimização dos recursos financeiros para o setor e um processo de gestão mais eficiente.

A finalidade da administração de materiais em qualquer empresa é de fazer chegar o material certo para a necessidade adequada no exato momento em que ele for necessário. Para que isto ocorra, é fundamental a criação de um banco de dados que gere informações capazes 
de apontar os estrangulamentos da demanda de consumo de material. No entanto, para a obtenção destas informações é importante planejar, controlar e organizar as necessidades, pois em geral os materiais devem ficar disponíveis em níveis adequados, evitar faltas e excessos que comprometam o capital do hospital e ainda resultar em medicamentos com prazos de validade vencidos, gerando altos custos para o Ministério da Saúde. Nas empresas voltadas para a área de saúde o cuidado com a gestão de estoque deverá ser ainda maior, uma vez que a falta poderá colocar em risco vidas humanas (FOGAÇA, 2006).

Em hospitais públicos, as limitações de ordem financeira, estrutural, físico, material e, de máquinas e equipamentos, fazem com que seus gestores ou servidores não utilizem "ferramentas modernas" da gestão de estoque. Sabendo que planejar e controlar custos são mecanismos que podem garantir a sobrevivência das instituições hospitalares, para a excelência do serviço é fundamental caminhar junto aos avanços tecnológicos. Muitos hospitais ainda registram seus dados em planilhas de Excel dificultando a administração e o gerenciamento.

Além disso, as instituições públicas contam, muitas vezes, com funcionários dedicados e responsáveis, porém pouco treinados para exercerem as tarefas inerentes às atividades ali desenvolvidas.

$\mathrm{Na}$ visão de CASTELLAR (1995), entende-se que a gestão de material, mais precisamente de estoques em materiais de uso hospitalar, precisa empenhar-se em proporcionar meios que permitam a melhoraria de sua eficiência e da qualidade dos seus serviços. Neste contexto, estão bons programas de controle de estoque, equipamentos como leitores em código de barras, treinamento de pessoal técnico, formação de equipes multiprofissionais e interdisciplinares, entre outras.

Outra grande dificuldade comum no contexto das instituições hospitalares públicas é a aquisição emergencial de materiais, sabendo que um aspecto importante da gestão de estoques em organizações de saúde, é o consumo em situações de emergência. As compras na rede pública são realizadas por licitação que se caracterizam por serem processos lentos e não satisfazem uma aquisição imediata.

Quando ocorre uma ruptura no estoque, além do desabastecimento por aumento de consumo ou falha na entrega, é comum cada supervisor ou gestor de unidade tender a criar seu próprio estoque de segurança, fora dos controles institucionais. Estes subestoques, pela falta de controle, nem sempre serão utilizados nas finalidades da unidade. O gestor de materiais de um hospital deve não apenas estabelecer políticas de estoque para condições normais de operação, mas também assegurar a capacidade do mesmo em atender a demanda emergencial.

Como citado anteriormente, no Estado do Paraná as aquisições são centralizadas em órgãos como o CEMEPAR e DELS, dificultando uma padronização própria da instituição. Segundo a International Organization for Standardization (ISO) (CUNHA, 1979), padronizar produtos abrigados em estoques é uma forma de normalização e auxilia na racionalização dos custos. Dentre as formas de racionalização, a padronização de medicamentos e material médico hospitalar é uma das soluções mais viáveis, pois procura definir o quê se deve manter em estoques. Racionalizar custos com medicamentos e material médico hospitalar implica em seguir normas técnicas que regulamentam o processo da assistência e aplicação de regras para o tratamento ordenado de uma atividade específica. Portanto, dentre as estratégias ligadas à gestão de estoque da farmácia hospitalar, torna-se fundamental a seleção dos produtos mais adequados ao perfil de utilização dos

mesmos pela instituição de saúde. 


\section{GESTÃO DE ESTOQUE NA FARMÁCIA DO HOSPITAL REGIONAL INFANTIL DOUTOR WALDEMAR MONASTIER EM CAMPO LARGO}

Em hospitais públicos, como há escassez de recursos, constitui-se de extrema importância o planejamento e a elaboração de mecanismos de controle capazes de melhorar a produtividade, a eficiência e a eficácia do serviço. A estratégia para a melhoria da qualidade do serviço está num planejamento o mais próximo possível da realidade, numa base de informações fidedignas, numa análise das possíveis causas de falhas e nas respostas geradas a partir desses elementos.

No Hospital Infantil Doutor Waldemar Monastier houve uma grande preocupação com a logística praticada pelo Estado o qual compromete o planejamento de abastecimento, gerando insatisfação da equipe interna - médicos, coordenadores, gestores e direção - e externos - os pacientes. Inserido neste contexto, foi criada uma comissão ou grupo de trabalho de suprimentos. Este trata-se de uma equipe multiprofissional, com a integração dos setores de Almoxarifado, Farmácia, área de Compras, Administração, Enfermagem, Nutrição e Hotelaria, subordinada a direção administrativa e com o objetivo principal de implantar uma eficiente gestão de suprimentos para o hospital.

Abordando conceitos teóricos de técnicas de administração em estoques, foram estabelecidos por meio da comissão, procedimentos para os almoxarifados, como técnicas de normalização: de estocagem, de controle, de classificação, padronização e codificação. As técnicas de normalização são essenciais para um efetivo planejamento e controle dos materiais utilizados no hospital. A normalização é importante porque indica o que o sistema assistencial irá necessitar, sendo, portanto, um dos elos de ligação entre a equipe de saúde e a farmácia. Foi implantada uma uniformização de procedimentos e impressos utilizados nos almoxarifados, de forma que todos os setores envolvidos trabalhem com o mesmo modelo, na execução de operações.

Nesta comissão adotaram-se parâmetros técnicos na gestão de estoques: consumo médio mensal, tempo de reposição, estoque de segurança, estoque mínimo. Além disso, foram determinadas regras para a aquisição de materiais, ouvindo sempre às chefias do almoxarifado e da farmácia, de forma a evitar compras mal dimensionadas.

Como se trata de um hospital novo, o Hospital Regional Infantil não possui indicadores de consumo, portanto, cada setor elaborou uma lista dos materiais necessários com a previsão de consumo mensal o qual foi designado internamente de "cardápio". Por meio deste, organizouse um fluxo para compras, de materiais não padronizados e não atendidos pelo DELS, em processos licitatórios, evitando às "compras de emergência".

Em decorrência do trabalho desta equipe multiprofissional, de colaboradores dedicados, diretores competentes, a gestão de suprimentos do Hospital Regional Infantil Doutor Waldemar Monastier, vem se aperfeiçoando a cada dia, por meio da construção de um sistema eficiente de planejamento e de um processo racional de aquisição.

Esta comissão de suprimentos é um trabalho contínuo e dinâmico que se reveste de valor especial, pois é um elo para que o hospital proporcione às crianças um atendimento seguro e com qualidade.

\section{CONCLUSÃO}

De maneira geral, as farmácias hospitalares são obrigadas a trabalhar com estoques altos com flutuações significativas e que abrigam uma grande diversidade de produtos, dificultando o planejamento de seu ressuprimento. Aliado a isso, tem-se os seguintes problemas: 
a maioria dos profissionais responsáveis por gerenciar estes estoques não possui qualificação adequada e muitas vezes o controle e as tomadas de decisão são realizadas sem o uso de sistemas computacionais específicos de suporte às tomadas de decisão. Levando-se em conta que o processo de produção do setor da saúde é muito complexo e o hospital constitui um centro de interação de várias disciplinas e profissões, incorporando tecnologias, gerando um modelo assistencial com grande variedade de itens e graus de diversidade.

No que diz respeito à logística, este estudo destacou que se constitui numa das maiores dificuldades da Administração Hospitalar Pública - a gestão de material devido à complexidade de atividades pertinentes à área, a saber: controle de estoque, compra, políticas assistenciais da saúde, entre outras.

Essas atividades envolvem, por parte do gestor, atributos como: planejamento, supervisão, delegação de tarefas, administração de conflitos, antecipação, poder decisório, comunicação, educação permanente com capacitação em serviço e criatividade.

Todo o trabalho do gestor farmacêutico, baseado no controle da qualidade, visa à satisfação das pessoas, seus colaboradores internos e externos, por isso se faz necessário que possa envolver a todos envolvidos no processo de suprimentos, como ocorre no Hospital Regional Infantil Doutor Waldemar Monastier, por meio da criação de um grupo de trabalho de suprimentos, para avaliar se os produtos e serviços atendem às necessidades e expectativas dos pacientes.

Desta forma, o paciente é um forte indicador de qualidade e produtividade, pois é ele quem demonstra a satisfação de suas necessidades, sendo a maior delas a preservação e manutenção de sua saúde, por meio de prestação de serviços médicos, laboratoriais, nutricionais, de limpeza e higiene e demais setores do hospital que expressem confiabilidade e garantia de qualidade dos produtos e serviços ofertados.

Esse estudo esboça, sucintamente, algumas reflexões sob uma visão crítica sobre o tema, sem a pretensão de esgotá-lo.

Espera-se ter contribuído para a Gestão Hospitalar no tocante à logística de suprimentos hospitalares e sugere a continuidade de pesquisas que discutam a referida problemática, no sentido de viabilizar economia, eficiência e qualidade na gestão pública de medicamentos e materiais para a saúde.

\section{REFERÊNCIAS:}

NOVAES, Mario Lucio de Oliveira; GONÇALVES, Antonio Augusto; SIMONETTI, Vera Maria Medina. Gestão das farmácias hospitalares através da padronização de medicamentos e utilização da curva ABC. XIII SIMPEP. São Paulo, 2006. p.3-8.

PAULUS JÚNIOR, Aylton. Gerenciamento de recursos materiais em unidades de saúde. Revista Espaço para a Saúde. Paraná, v.7, n.1, p. 30-45, 2005.

YUK, Caroline Silva; KNEIPP, Jordana Marques; MAEHLER, Alisson Eduard.

Sistemática de distribuição de medicamentos em organizações hospitalares. XV Congresso de Iniciação Científica. Universidade Federal de Pelotas.Disponívelem:http://www. ufpel.edu.br/xivcic/arquivos/conteudo_SA.html

BALLOU, Ronald H. Logística empresarial: transportes, administração de materiais e distribuição física. São Paulo: Atlas, 1993. 388p.

FOGAÇA, Moacir. Administração de logística: ênfase nos processos hospitalares. Apostila 
do Curso de Pós-Graduação Lato Sensu da Escola de Saúde Pública. Santa Catarina, 2006. Disponível em:

http://www.saude.sc.gov.br/admin_ses/diretoria_desenv_humano/escola_saude_publica/ materiais/prof_Moacir\%C2\%AD_Fogaca/APOSTILA\%20TEXXTO\%20SA\%C3\%9ADE.doc.

SENGE, P., ROSS, R., SMITH, B., ROBERTS, C., KLEINER, A. A quinta disciplina - caderno de campo: estratégias e ferramentas para construir uma organização que aprende. Rio de Janeiro: Qualitymark, 1995.

BALLOU, Ronald H. Gerenciamento da cadeia de suprimentos / logística empresarial. 5. ed. Porto Alegre: Bookman, 2006.

MAUDONNET, Renato. Administração Hospitalar, Rio de Janeiro. Editora Cultura Médica, 1988.

BERTAGLIA, Paulo Roberto. Logística e Gerenciamento da Cadeia de Abastecimento, São Paulo: Saraiva 2005.

Lei 8.666 de 21 de junho de 1993. Regulamenta o art. 37, inciso XXI, da Constituição Federal, institui normas para licitações e contratos da Administração Pública. Diário Oficial da União de 06 de jun., 1993. Brasília, 1993.

Lei 8.883 de 8 de junho de 1994. Altera dispositivos da Lei $n^{\circ} \mathbf{8 . 6 6 6}$, de 21 de junho de 1993, que regulamenta o art. 37, inciso XXI, da Constituição Federal, institui normas para licitações. Diário Oficial da União. Brasília, 1994.

Lei 9648 de 27 de maio de 1998. Altera dispositivos das Leis $n^{\circ} 3.890-A$, de 25 de abril de $1961, n^{\circ} 8.666$, de 21 de junho de 1993, $n^{\circ} 8.987$, de 13 de fevereiro de 1995, $n^{\circ} 9.074$, de 7 de julho de 1995, n 9.427, de 26 de dezembro de 1996. Diário Oficial da União. Brasília, 1998.

CASTELAR, Rosa Maria; MORDELET Pratrick; GRABOIS Victor. Gestão Hospitalar: um desafio para o hospital brasileiro. Paris: Editions École Nationale de la Santé Publique, 1995.

SANTICH, I. R., ROJAS, C. M. Curso de administração de sistemas de abastecimento de medicamentos essenciais. Mod. 7, armazenamento e distribuição de medicamentos essenciais. Brasília:OPAS/OMS, 1994. 89 p.

SANTICH, I. R., GALLI, A. La asistencia farmacéutica en el Sistema Único de Salud de Ceará.; elementos para su transformación. Fortaleza: Esc. De Saúde pública do Ceará, 1995. 192 p.

CUNHA, G.W.B. Padronização de medicamentos na área hospitalar. In: CONGRESSO DE ADMINISTRAÇÃO HOSPITALAR, 1979, São Paulo.

BRASIL. Portaria ${ }^{\circ}{ }^{1}$ 1.818, de 02 de dezembro de 1997, da Secretaria Nacional de Vigilância Sanitária.

SESA - SECRETARIA DE ESTADO DA SAÚDE DO PARANÁ, Curitiba, www.saude.pr.gov.br, Abril, 2010. 